\title{
Non-linear System Identification for Aeroelastic Systems with Application to Experimental Data
}

\author{
Sunil L. Kukreja* \\ NASA Dryden Flight Research Center, Edwards, California, 93523-0273, USA
}

\begin{abstract}
Representation and identification of a non-linear aeroelastic pitch-plunge system as a model of the NARMAX class is considered. A non-linear difference equation describing this aircraft model is derived theoretically and shown to be of the NARMAX form. Identification methods for NARMAX models are applied to aeroelastic dynamics and its properties demonstrated via continuous-time simulations of experimental conditions. Simulation results show that (i) the outputs of the NARMAX model match closely those generated using continuous-time methods and (ii) NARMAX identification methods applied to aeroelastic dynamics provide accurate discrete-time parameter estimates. Application of NARMAX identification to experimental pitchplunge dynamics data gives a high percent fit for cross-validated data.
\end{abstract}

\section{Introduction}

SYstem identification or mathematical modeling is the process of developing or improving a mathematical repreSentation of a physical system based on observed data. System identification is a critical step in aircraft development, analysis and validation for flight worthiness.

One such application of system identification in the flight-test community is for the analysis of aeroelasticity. The analysis of aeroelasticity is concerned with the interaction of inertial, structural and aerodynamic forces ${ }^{1}$ Previous approaches have modelled aeroelasticity with linear time-invariant (LTI) models. These linear models have been successful in providing approximate estimates of an aircraft's response to gust, turbulence and external excitations. However, when aircraft speeds increase to high subsonic or transonic Mach numbers linear models no longer provide accurate predictions of the aircraft's behavior. Some of the behavior that cannot be modelled linearly includes transonic dip, air flow separation and shock oscillations which can induce non-linear phenomena such as limit cycle oscillations (LCO) $\sqrt[23]{3}$ The onset of LCOs has been observed on several aircraft such as the F-16C or F/A-18 and cannot be modelled properly as a LTI system. ${ }^{4}$ This has necessitated the application of non-linear identification techniques to accurately model LCO dynamics.

Over the past several decades, significant achievements have been made in several areas of nonparametric nonlinear system identification (see e.g 5 .5677). Recent work in the aerospace community has attempted to address these non-linear aeroelastic phenomena using Volterra kernel methods. ${ }^{8}$ These methods provide a convenient means of characterising LCOs but suffer from a highly over-parameterised model description and do not lend themselves to efficient control synthesis.

Parametric representations of non-linear systems typically contain a small number of coefficients which can be varied to alter the behavior of the equation and may be linked to the underlying system. Leontaritis and Billings 59.10 have proposed the NARMAX (Non-linear AutoRegressive, Moving Average eXogenous) structure as a general parametric form for modeling non-linear systems. NARMAX models describe non-linear systems in terms of linear-inthe-parameters difference equations relating the current output to (possibly non-linear) combinations of inputs and past outputs. It is suitable for modeling both the stochastic and deterministic components of a system and is capable of describing a wide variety of non-linear systems $\underline{11 \mid 12}$ This formulation yields compact model descriptions that may be readily identified and may afford greater interpretability. NARMAX models have been successfully demonstrated for modelling the input-output behavior of many complex systems such as ones found in engineering and biology 13,14

Currently, development and test of aircraft takes many years and considerable expenditure of limited resources. One reason for lengthy development time/costs is many models (and hence control strategies) need to be developed throughout the flight envelope. The power of parametric non-linear identification techniques (i.e. NARMAX models)

${ }^{*}$ Aerospace Research Engineer, Structural Dynamics Group, NASA Dryden Flight Research Center. 
is that they can describe complex aeroelastic behavior over a large operating range. Consequently, this provides models that can be more robust and reduce development time.

Although the NARMAX structure is well suited to modeling the input-output behavior of a aeroelastic system, to date it has not been investigated by the flight-test community. Therefore, in this paper, we (i) theoretically analyse a non-linear pitch-plunge model of aircraft dynamics to derive its NARMAX representation, (ii) assess the applicability of this non-linear model for the identification of aerospace systems and (iii) investigate the suitability of NARMAX identification methods applied to aircraft dynamics.

Our results show that the NARMAX model class provides an ideal framework for modeling the input-output behavior of a non-linear pitch-plunge model describing aircraft dynamics. Identification results illustrate that methods for identification of NARMAX models are well suited for identifying aircraft dynamics. Analysis of experimental data using NARMAX identification techniques provides a parameter set that explains the input-output data well. Overall, this paper contributes to the understanding of the use of parametric identification techniques for modeling of aerospace systems.

The organisation of this paper is as follows. The NARMAX model structure is described in \$II In $\$$ III a continuous-time representation of a non-linear pitch-plunge model describing aircraft dynamics is given, whilst its NARMAX representation is derived in $\$[$ IV] Section V illustrates the results of simulating this NARMAX representation of pitch-plunge dynamics. In $\$$ VI the applicability of NARMAX identification to this model representation via simulations of experimental condition is assessed. Section VII presents the results of identifying experimental wind tunnel data and $\$[\mathrm{VIII}$ provides a discussion of the major finds. Lastly, in $\$[\mathrm{IX}$ conclusions and significance of the results are given.

\section{NARMAX Model}

The NARMAX (Non-linear AutoRegressive, Moving Average eXogenous) structure is a general parametric form for modeling non-linear systems. 9 This structure describes both the stochastic and deterministic components of nonlinear systems. Many non-linear systems are a special case of the general NARMAX structure. ${ }^{12}$ The NARMAX structure models the input-output relationship as a non-linear difference equation of the form

$$
z(n)=\mathscr{F}^{l}\left[z(n-1), \cdots, z\left(n-n_{z}\right), u(n), \cdots, u\left(n-n_{u}\right), e(n-1), \cdots, e\left(n-n_{e}\right)\right]+e(n) .
$$

$\mathscr{F}^{l}$ denotes a non-linear mapping, $u$ is the controlled or exogenous input, $z$ is the measured output, and $e$ is the uncontrolled input or innovation. The non-linear mapping, $\mathscr{F}^{l}$, can be described by a wide variety of non-linear functions such as a $\tanh (\cdot)$ or splines (i.e. hard non-linearities) ${ }^{11 \mid 12}$ For simplicity, non-linearities are only considered that can be described by a polynomial expansion. This class of non-linear difference equations describes the dynamic behavior of a system as a linear and/or non-linear expansion of the input, output and error. It may include a variety of non-linear terms, such as terms raised to an integer power (e.g., $u^{3}(n-5)$ ), products of present and past inputs (e.g., $u(n) u(n-3)$ ), past outputs (e.g., $\left.z^{2}(n-2) z(n-7)\right)$, or cross-terms (e.g., $u(n-3) z^{2}(n-4)$ ). This system description encompasses many forms of non-linear difference equations that are linear-in-the-parameters. Thus, there are no problems with local minima.

\section{Non-linear Pitch-Plunge Model of Aircraft Dynamics}

O'Neil et al. $!^{15 \mid 16}$ developed a non-linear pitch-plunge model (Fig. 11) describing aircraft aeroelastic dynamics. Figure 1 characterises aeroelastic wing dynamics for experiments performed on the Texas A\& M testbed 16 This model provides a relationship between control surface deflection as input and pitch-plunge displacement and velocity as outputs of a single-input multiple-output non-linearity followed by a simple integrator.

The model presented in Fig. 1 has been derived from the governing equations of motion for aeroelastic systems as

$$
\left[\begin{array}{cc}
m & m x_{\alpha} b \\
m x_{\alpha} b & I_{\alpha}
\end{array}\right]\left[\begin{array}{c}
\ddot{h} \\
\ddot{\alpha}
\end{array}\right]+\left[\begin{array}{cc}
c_{h} & 0 \\
0 & c_{\alpha}
\end{array}\right]\left[\begin{array}{c}
\dot{h} \\
\dot{\alpha}
\end{array}\right]+\left[\begin{array}{cc}
k_{h} & 0 \\
0 & k_{\alpha}(\alpha)
\end{array}\right]\left[\begin{array}{c}
h \\
\alpha
\end{array}\right]=\left[\begin{array}{c}
-L \\
M
\end{array}\right]
$$

where $h$ denotes plunge motion, $\alpha$ pitch angle, $x_{\alpha}$ nondimensional distance between elastic axis and center of mass, $m$ wing mass, $I_{\alpha}$ mass moment of inertia of the wing about the elastic axis, $b$ semichord of the wing, $\left\{c_{h}, c_{\alpha}\right\}$ plunge and pitch structural damping coefficients, $\left\{k_{h}, k_{\alpha}\right\}$ plunge and pitch structural spring constants, and $L, M$ aerodynamic lift and moment. 


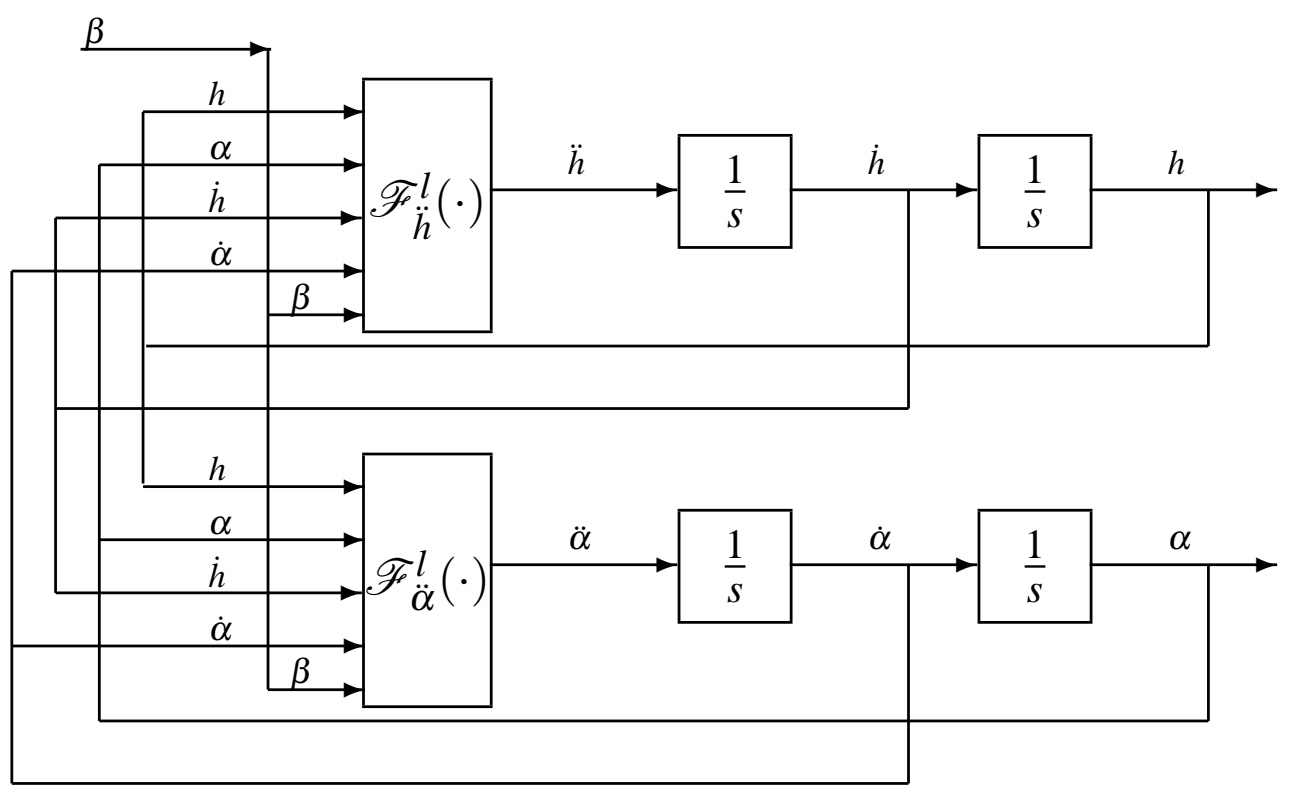

Figure 1. System structure assumed for modeling and identification of pitch-plunge aeroelastic dynamics.

Typically, quasi-steady aerodynamic forces and moments are assumed which can be modeled as

$$
\begin{aligned}
L & =\rho U^{2} b c_{l_{\alpha}}\left[\alpha+\frac{\dot{h}}{U}+\left(\frac{1}{2}-a\right) b \frac{\dot{\alpha}}{U}\right]+\rho U^{2} b c_{l_{\beta}} \beta \\
M & =\rho U^{2} b^{2} c_{m_{\alpha}}\left[\alpha+\frac{\dot{h}}{U}+\left(\frac{1}{2}-a\right) b \frac{\dot{\alpha}}{U}\right]+\rho U^{2} b^{2} c_{m_{\beta}} \beta
\end{aligned}
$$

where $\rho$ denotes density of air, $U$ free-stream velocity, $c_{m_{\alpha}}, c_{l_{\alpha}}$ moment and lift coefficients per angle of attack, $c_{m_{\beta}}, c_{l_{\beta}}$ moment and lift coefficient per control surface deflection, $\beta$ control surface deflection, and $a$ nondimensional distance from midchord to elastic axis.

Although, several classes of non-linear mappings for stiffness contributions $k_{\alpha}(\alpha)$ have been investigated for open loop dynamics of aeroelastic systems,,$\frac{17,18,19 \mid 20}{2}$ the work of O'Neil et al ${ }^{15 \mid 16}$ demonstrated that a polynomial mapping of the form

$$
k_{\alpha}(\alpha)=k_{\alpha_{0}}+k_{\alpha_{1}} \alpha+k_{\alpha_{2}} \alpha^{2}+k_{\alpha_{3}} \alpha^{3}+k_{\alpha_{4}} \alpha^{4}
$$

describes the behavior of this testbed well.

Equations of motion are derived by combining Eqns. 2 and 3 to yield

$$
\begin{aligned}
{\left[\begin{array}{cc}
m & m x_{\alpha} b \\
m x_{\alpha} b & I_{\alpha}
\end{array}\right]\left[\begin{array}{c}
\ddot{h} \\
\ddot{\alpha}
\end{array}\right] } & +\left[\begin{array}{cc}
c_{h}+\rho U b c_{l_{\alpha}} & \rho U b^{2} c_{l_{\alpha}}\left(\frac{1}{2}-a\right) \\
\rho U b^{2} c_{m_{\alpha}} & c_{\alpha}-\rho U b^{3} c_{m_{\alpha}}\left(\frac{1}{2}-a\right)
\end{array}\right]\left[\begin{array}{c}
\dot{h} \\
\dot{\alpha}
\end{array}\right] \\
+ & {\left[\begin{array}{cc}
k_{h} & \rho U^{2} b c_{l_{\alpha}} \\
0 & -\rho U^{2} b^{2} c_{m_{\alpha}}+k_{\alpha}(\alpha)
\end{array}\right]\left[\begin{array}{c}
h \\
\alpha
\end{array}\right]=\left[\begin{array}{c}
-\rho b c_{l_{\beta}} \\
\rho b^{2} c_{m_{\beta}}
\end{array}\right] U^{2} \beta . }
\end{aligned}
$$

The model form presented in Fig. 1 is derived by transforming Eqn. 5 to give

$$
\dot{\mathbf{x}}=\mathbf{f}_{\mu}(\mathbf{x})+\mathbf{g}(\mathbf{x}) \mu \beta
$$

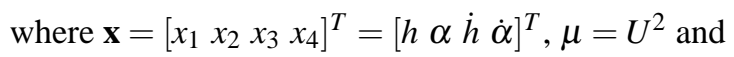

$$
\mathbf{f}_{\mu}=\left[\begin{array}{c}
x_{3} \\
x_{4} \\
-k_{1} x_{1}-\left(k_{2} \mu+p\left(x_{2}\right)\right) x_{2}-c_{1} x_{3}-c_{2} x_{4} \\
-k_{3} x_{1}-\left(k_{4} \mu+q\left(x_{2}\right)\right) x_{2}-c_{3} x_{3}-c_{4} x_{4}
\end{array}\right], \quad \mathbf{g}(\mathbf{x})=\left[\begin{array}{c}
0 \\
0 \\
g_{3} \\
g_{4}
\end{array}\right] .
$$


The supplementary variables $k_{i}, i=1,2,3,4$ and $g_{j}, j=3,4$ are provided in Table 1 in relationship to the aeroelastic parameters given in Eqns. 2 and 3

\begin{tabular}{c|c}
\hline Supplementary Variable & Relationship to System Parameters \\
\hline$d$ & $m\left(I_{\alpha}-m x_{\alpha}^{2} b^{2}\right)$ \\
$k_{1}$ & $\frac{I_{\alpha} k_{h}}{d}$ \\
$k_{2}$ & $\frac{I_{\alpha} \rho b c_{\alpha}+m x_{\alpha} b^{3} \rho c_{m_{\alpha}}}{d}$ \\
$k_{3}$ & $\frac{-m x_{\alpha} b k_{h}}{d}$ \\
$k_{4}$ & $\frac{-m x_{\alpha} b^{2} \rho c_{l_{\alpha}}-m \rho b^{2} c_{m \alpha}}{d}$ \\
$c_{1}$ & $\frac{I_{\alpha}\left(c_{h}+\rho U b c_{l_{\alpha}}\right)+m x_{\alpha} \rho U b^{3} c_{m_{\alpha}}}{d}$ \\
$c_{2}$ & $\frac{-m x_{\alpha} b c_{h}-m x_{\alpha} \rho U b^{2} c_{l_{\alpha}}-m \rho U b^{2} c_{m \alpha}}{d}$ \\
$c_{3}$ & $\left.\frac{m c_{\alpha}-m x_{\alpha} \rho U b^{3} c_{l_{\alpha}}\left(\frac{1}{2}-a\right)-m \rho U b^{3} c_{m_{\alpha}}\left(\frac{1}{2}-a\right)}{d}-a\right)-m x_{\alpha} b c_{\alpha}+m x_{\alpha} \rho U b^{4} c_{m_{\alpha}}\left(\frac{1}{2}-a\right)$ \\
$c_{4}$ & $\frac{-I_{\alpha} \rho b c_{l_{\beta}}-m x_{\alpha} b^{3} \rho c_{m_{\beta}}}{d}$ \\
$g_{3}$ & $\frac{m x_{\alpha} b^{2} \rho c_{l_{\beta}}+m \rho b^{2} c_{m_{\beta}}}{d}$ \\
$g_{4}$ & $\frac{-m x_{\alpha} b}{d} k_{\alpha}\left(x_{2}\right)$ \\
$p\left(x_{2}\right)$ & $\frac{m}{d} k_{\alpha}\left(x_{2}\right)$ \\
$q\left(x_{2}\right)$ &
\end{tabular}

Table 1. Supplementary system variables.

Transformation of Eqn. 5 to Eqn. 6 and introduction of supplementary variables (Table 1 ) provides the simple model description presented in Fig. 11. The non-linear mappings for this model description are given as

$$
\begin{aligned}
\mathscr{F}_{\ddot{a}}^{l}(\cdot)= & -k_{3} h(n)\left[k_{4} \mu \frac{m}{d}\left(k_{\alpha_{0}}+k_{\alpha_{1}} \alpha+k_{\alpha_{2}} \alpha^{2}+k_{\alpha_{3}} \alpha^{3}+k_{\alpha_{4}} \alpha^{4}\right)\right] \alpha(n)-c_{3} \dot{h}(n) \\
& -c_{4} \dot{\alpha}(n)+g_{4} \mu U(n) \\
= & -b_{1} h(n)-\left[b_{2} \alpha(n)+b_{3} \alpha^{2}(n)+b_{4} \alpha^{3}(n)+b_{5} \alpha^{4}(n)+b_{6} \alpha^{5}(n)\right]-b_{7} \dot{h}(n) \\
& -b_{8} \dot{\alpha}(n)+b_{9} U(n) \\
= & \ddot{\alpha}(n) \\
\mathscr{F}_{\ddot{h}}^{l}(\cdot)= & -k_{1} h(n)-\left[k_{2} \mu+\frac{-m x_{\alpha} b}{d}\left(k_{\alpha_{0}}+k_{\alpha_{1}} \alpha+k_{\alpha_{2}} \alpha^{2}+k_{\alpha_{3}} \alpha^{3}+k_{\alpha_{4}} \alpha^{4}\right)\right] \alpha(n) \\
& -c_{1} \dot{h}(n)-c_{2} \dot{\alpha}(n)+g_{3} \mu U(n) \\
= & -a_{1} h(n)-\left[a_{2} \alpha(n)+a_{3} \alpha^{2}(n)+a_{4} \alpha^{3}(n)+a_{5} \alpha^{4}(n)+a_{6} \alpha^{5}(n)\right]-a_{7} \dot{h}(n) \\
& -a_{8} \dot{\alpha}(n)+a_{9} U(n) \\
= & \ddot{h}(n)
\end{aligned}
$$

Note this system (Fig. 1) can be described in terms of pitch-plunge displacement or velocity. Here pitch-plunge is chosen in terms of velocity because (i) it offers a model description with lower dynamic order and (ii) velocity feedback models are often used for vibration suppression.

\section{Discrete-Domain Approximations}

Many methods exist for discretisation of continuous-time systems/signals. Most commonly used are Euler's forward, Euler's backward or Tustin's method (also known as the bilinear transformation method), see e.g. ${ }^{[1 \mid 22]}$ Each has its

$$
4 \text { of } 13
$$


advantages as well as disadvantages. Whilst, Tustin's and Euler's backward methods provide a superior approximation to a continuous-time signal the goal in system identification is not (directly) signal reproduction but model estimation.

Tustin's method provides excellent signal estimation but its use for modeling a pure integrator yields system descriptions which can be overly complex, e.g. redundant terms. Euler's backward method also provides good signal estimation but provides a model that is not intuitive. Models based on this approximation would include the current output as one of the model terms, leading to an algebraic loop. Although Euler's forward method is well known to be unstable, this is only true if the sampling rate is not sufficiently large. For identification purposes this does not pose a concern since the signals need to be sampled "at least" twice Nyquist and, hence, stability is achieved. Generally, the rule of thumb is to sample a signal at least 4-10 times the highest known (or suspected) system dynamics. ${ }^{23}$ For the pitch-plunge system under investigation, Euler's forward method provides a model description that is both stable and intuitive. Moreover, all three methods converge to similar accuracies for sufficiently large sampling rates. For these reasons Euler's forward method is chosen to model the system dynamics.

\section{Theoretical Analysis}

The pitch-plunge model is given in continuous-time. This section shows how the model can be converted to discrete-time and rewritten as a NARMAX model. To do so, note that the two non-linearities can be decoupled and analysed separately since they yield two separate model descriptions for pitch and plunge velocity.

Euler's forward (explicit) method 21

$$
\frac{1}{s}=\dot{x}(0)+\int_{0}^{t} \ddot{x}(t) d t \approx \dot{x}(n-1)+T \ddot{x}(n-1)
$$

where $T$ is the sample time, was used to approximate the continuous-time integrator, where $\ddot{x}$ is replaced by $\ddot{\alpha}$ and $\ddot{h}$ for pitch and plunge, respectively.

The non-linearities used for this analysis, to derive an input-output model of pitch and plunge, were given in Eqns. 8 and 9 . In addition, the models are assumed corrupted by output additive (measurement) noise as

$$
\begin{aligned}
\dot{\alpha}(n) & =\dot{\alpha}_{n f}(n)+e_{\dot{\alpha}}(n) \\
\dot{h}(n) & =\dot{h}_{n f}(n)+e_{\dot{h}}(n)
\end{aligned}
$$

where $\dot{\alpha}(n)$ and $\dot{h}(n)$ are the noise corrupted outputs, $\dot{\alpha}_{n f}(n)$ and $\dot{h}_{n f}(n)$ the unmeasured noise free outputs and $e_{\dot{\alpha}}(n)$ and $e_{\dot{h}}(n)$ the measurement noise.

After collecting terms and combining, the overall non-linear models were represented as non-linear difference equations with 10 terms each as

$$
\begin{aligned}
\dot{\alpha}(n) & =\gamma_{1} \dot{\alpha}(n-1)+\gamma_{2} h(n-1)+\gamma_{3} \alpha(n-1)+\gamma_{4} \alpha(n-1)^{2}+\gamma_{5} \alpha(n-1)^{3} \\
& +\gamma_{6} \alpha(n-1)^{4}+\gamma_{7} \alpha(n-1)^{5}+\gamma_{8} \dot{h}(n-1)+\gamma_{9} u(n-1)+\gamma_{10} e_{\dot{\alpha}}(n-1)+e_{\dot{\alpha}}(n) \\
\dot{h}(n) & =\theta_{1} \dot{h}(n-1)+\theta_{2} h(n-1)+\theta_{3} \alpha(n-1)+\theta_{4} \alpha(n-1)^{2}+\theta_{5} \alpha(n-1)^{3} \\
& +\theta_{6} \alpha(n-1)^{4}+\theta_{7} \alpha(n-1)^{5}+\theta_{8} \dot{\alpha}(n-1)+\theta_{9} u(n-1)+\theta_{10} e_{\dot{h}}(n-1)+e_{\dot{h}}(n) .
\end{aligned}
$$

These are NARMAX models since (i) they include input-output terms that are combinations of linear and non-linear integer powers and (ii) are linear-in-the-parameters. Table 2 shows the relationship of discrete-time NARMAX parameters in (13) to the underlying continuous-time coefficients.

\section{Validation of NARMAX Pitch-Plunge Model}

The accuracy of this system representation was validated by simulating the pitch-plunge model in continuous-time using Simulink (Fig. 1 1). The non-linearities used in this continuous-time simulation were the fifth-order power series described in Eqns. 8 and 9. The parameters used in the simulation were typical values found in experiments and are given in Table $3{ }^{15}$ The system was excited using a $5 \mathrm{~Hz}$ chirp input.

\section{V.A. Output Accuracy}

To determine the validity of this NARMAX description model (13), its response is simulated for a parameter set corresponding to those used for the continuous-time model. The input sequence was a $5 \mathrm{~Hz}$ chirp with a signal 


\begin{tabular}{cccc}
\hline $\begin{array}{c}\text { NARMAX } \\
\text { Plunge } \\
\text { Coefficient }\end{array}$ & Relationship to & $\begin{array}{c}\text { NARMAX } \\
\text { Pitch }\end{array}$ & Relationship to \\
\hline$\theta_{1}$ & $1-T a_{7}$ & $\gamma_{1}$ & $1-T b_{8}$ \\
$\theta_{2}$ & $T a_{1}$ & $\gamma_{2}$ & $T b_{1}$ \\
$\theta_{3}$ & $T a_{2}$ & $\gamma_{3}$ & $T b_{2}$ \\
$\theta_{4}$ & $T a_{3}$ & $\gamma_{4}$ & $T b_{3}$ \\
$\theta_{5}$ & $T a_{4}$ & $\gamma_{5}$ & $T b_{4}$ \\
$\theta_{6}$ & $T a_{5}$ & $\gamma_{6}$ & $T b_{5}$ \\
$\theta_{7}$ & $T a_{6}$ & $\gamma_{7}$ & $T b_{6}$ \\
$\theta_{8}$ & $T a_{8}$ & $\gamma_{8}$ & $T b_{7}$ \\
$\theta_{9}$ & $T a_{9}$ & $\gamma_{9}$ & $T b_{9}$ \\
$\theta_{10}$ & $-\left(1-T a_{7}\right)$ & $\gamma_{10}$ & $-\left(1-T b_{8}\right)$ \\
\hline
\end{tabular}

Table 2. Theoretical relationship of NARMAX model parameter set to continuous-time system coefficients.

\begin{tabular}{cc}
\hline CT Coefficient & Value \\
\hline$b$ & $0.135 \mathrm{~m}$ \\
span & $0.600 \mathrm{~m}$ \\
$k_{h}$ & $2844.4 \mathrm{~N} / \mathrm{m}$ \\
$c_{h}$ & $27.43 \mathrm{~N} \mathrm{~s} / \mathrm{m}$ \\
$\rho$ & $1.225 \mathrm{~kg} / \mathrm{m}^{3}$ \\
$c_{l_{\alpha}}$ & $6.28 / \mathrm{rad}$ \\
$c_{l_{\beta}}$ & $3.358 / \mathrm{rad}$ \\
$c_{m_{\alpha}}$ & $(0.5+a) c_{l_{\alpha}} / \mathrm{rad}$ \\
$c_{m_{\beta}}$ & $-0.635 / \mathrm{rad}$ \\
\hline
\end{tabular}

Table 3. Continuous-time system coefficients.

duration of 30 seconds. The chirp input had an operating range between $\pm 1.0 \mathrm{rad}$ (see upper panel of Fig. 2).

Using a fifth-order non-linearity, the frequency content of the signal at the output of the non-linearity will be at least $25 \mathrm{~Hz}$ (five times the $5 \mathrm{~Hz}$ chirp signal). To avoid internal aliasing, a sampling rate of $100 \mathrm{~Hz}(T=0.01 \mathrm{~s})$ is selected, 4 times greater than the internal $25 \mathrm{~Hz}$ signal.

The simulated output $(\hat{y}(n))$ of the NARMAX description model was compared with the output of the continuoustime simulation $(y(n))$ by computing the percent variance accounted for by the NARMAX model as the percent quality of fit $(\% \mathrm{QF})$ :

$$
\% Q F=\left(1-\frac{\frac{1}{N} \sum_{n=1}^{N}(y(n)-\hat{y}(n))^{2}}{\frac{1}{N} \sum_{n=1}^{N}(y(n))^{2}}\right) \times 100,
$$

where $N$ is the record length.

\section{V.B. Simulation Result}

This subsection illustrates the results of simulating the pitch-plunge model in continuous-time against the discretetime NARMAX predictions. Fig. 2 shows the simulation input (upper panel) and predicted velocity outputs of the NARMAX description models superimposed on top of the continuous-time outputs of the pitch (lower left panel) and

$$
6 \text { of } 13
$$


plunge (lower right panel) velocity models. With over $99 \%$ QF the NARMAX outputs matched that of the continuous-

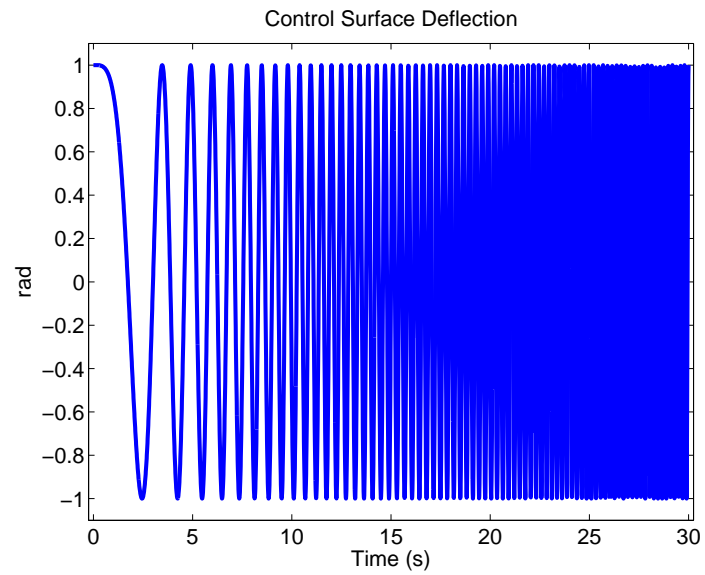

(a)

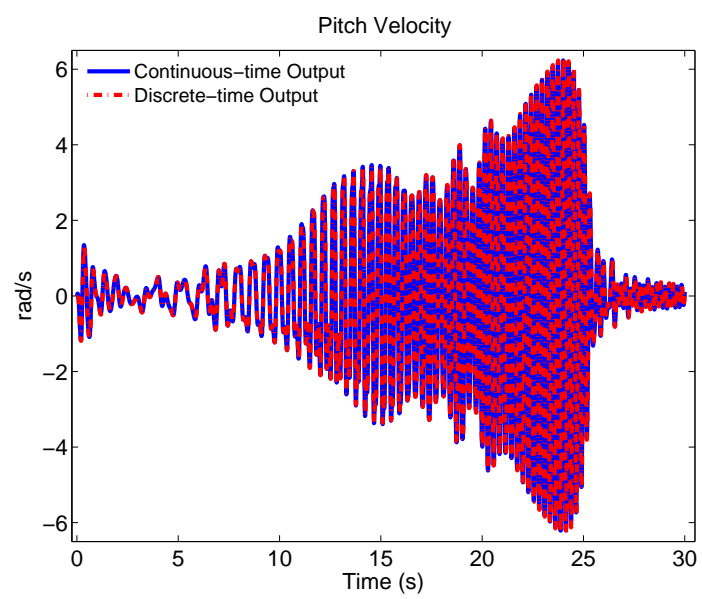

(b)

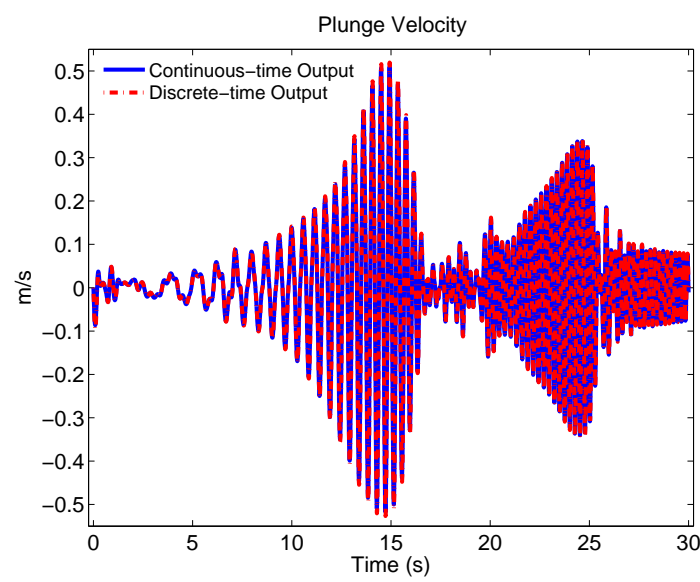

(c)

Figure 2. Upper: Input to simulated pitch-plunge model in continuous-time and NARMAX description model. Lower: Output of simulated pitch-plunge velocity model in continuous-time and NARMAX description model: (b) Pitch velocity; (c) Plunge velocity. Note that the two outputs are almost identical.

time simulation with negligible error.

\section{NARMAX Identification of Pitch-Plunge Model}

We then assessed the utility of methods developed for identifying NARMAX models using sampled data from this continuous-time simulation. An extended least-squares (ELS) algorithm 24 25:26 was used to identify model parameters.

The NARMAX description of the pitch-plunge velocity models (13) are described by past outputs which are linear-in-the-parameters. In the presence of output additive noise (12) and 13), these terms result in lagged values of disturbance terms which are also linear-in-the-parameters. If these lagged errors are not modeled they induce a bias in the parameter estimates ${ }^{27.28} 22$ The ELS algorithm was implemented because it is designed to model lagged error terms thereby providing unbiased parameter estimates.

It is well known that ELS may suffer from convergence problems ${ }^{29,22] 30}$ However, no prediction error identification (PEI) method is optimal. For the pitch-plunge velocity models, this is deemed the best estimation technique because it provides an unbiased estimate of model parameters. ${ }^{[2]}$ Other estimation techniques such as maximum like- 
lihood (ML), instrumental variables (IV), weighted least-squares (WLS), etc., are difficult to implement and also have convergence problems $22,30,31$ For this reason ELS is chosen.

\section{VI.A. Analysis of NARMAX Model Parameters}

A Monte-Carlo study of these NARMAX parameters (Eqn. 13 and Table 2) was performed to assess their accuracy and variability using the ELS estimator. One thousand Monte-Carlo simulations were generated in which the input-output realisation was the same but had a unique Gaussian white, zero-mean, noise sequence added to the output. The output additive noise amplitude was increased in increments of $5 \mathrm{~dB}$, from 20 to $0 \mathrm{~dB}$ SNR. Parameter mean and standard deviation was computed from the one thousand estimates. The input used for this study was the same $5 \mathrm{~Hz}$ chirp signal described in $\$$ V.A.

For this study, the system order and structure were assumed to be known with the coefficient set in (13) and Tables 2 and 3 . The regressor matrix used by this algorithm was formed to contain only those columns (parameters) that corresponded to the theoretical analysis $(13)$. It is reasonable to assume that the order and structure are known because the goal is to identify a model in the model class described by (13).

In experimental settings often only pitch-plunge displacement and/or acceleration signals are available. For a velocity model description, the velocity signal is required for identification. Therefore, pitch-plunge acceleration signals are numerically integrated to obtain a velocity profile. The estimation set consisted of $N=3,000$ data points sampled at $T=0.01 \mathrm{~s}$. The estimated parameters were cross-validated with a fresh noise corrupted output to compute the $\% \mathrm{QF}$ of the predicted pitch and plunge velocity. The validation set consisted of $N_{v}=3,000$ data points 22 32

\section{VI.B. Identification Results of Simulated Model}

Fig. 3 shows the results of identifying this simulated model of pitch-plunge. The NARMAX parameters in this figure correspond to those given in Table 2. This figure shows that the identified parameter values corresponded closely to those derived theoretically for all SNRs. Note that it is not expected that the mean value of parameters $\gamma_{10}$ and $\theta_{10}$ be close to the theoretically computed value since they correspond to lagged error terms. Lagged error terms are difficult to identify accurately even with high SNR since they model the output additive noise which is an unmeasurable stochastic process. This stochastic process is modeled (approximated) by a deterministic signal of prediction errors which is only a (poor) estimate of the noise 2822

Fig. 4 presents a result of cross-validation for a typical parameter set for this study. The top panels shows a noise corrupted output used for identification and the bottom panels show a predicted output superimposed on top of the noise free output. The predicted output matched the measured output with over $98 \% \mathrm{QF}$.

\section{Identification of Experimental Pitch-Plunge Data}

Lastly, the identification technique is assessed on experimental wing section data collected in the wind tunnel at the Department of Aerospace Engineering (Texas A\&M University) by the Aeroelasticity Research Group. The data analysed for this study does not contain a flap control input but instead had an initial condition associated with plunge displacement. Data with a control input was unavailable for analysis.

The velocity model descriptions given in Eqn. 13 are in terms of a control input and zero initial conditions. For the present study the model was modified to reflect a lack of exogenous input and the presence of an initial condition. Model 13 is reformulated as

$$
\begin{aligned}
\dot{h}(n) & =\theta_{1} \dot{h}(n-1)+\theta_{2} h(n-1)+\theta_{3} \alpha(n-1)+\theta_{4} \alpha(n-1)^{2}+\theta_{5} \alpha(n-1)^{3} \\
& +\theta_{6} \alpha(n-1)^{4}+\theta_{7} \alpha(n-1)^{5}+\theta_{8} \dot{\alpha}(n-1)+\theta_{9} \delta(n)+\theta_{10} e_{\dot{h}}(n-1)+e_{\dot{h}}(n) \\
\dot{\alpha}(n) & =\gamma_{1} \dot{\alpha}(n-1)+\gamma_{2} h(n-1)+\gamma_{3} \alpha(n-1)+\gamma_{4} \alpha(n-1)^{2}+\gamma_{5} \alpha(n-1)^{3} \\
& +\gamma_{6} \alpha(n-1)^{4}+\gamma_{7} \alpha(n-1)^{5}+\gamma_{8} \dot{h}(n-1)+\gamma_{9} e_{\dot{\alpha}}(n-1)+e_{\dot{\alpha}}(n)
\end{aligned}
$$

where $\delta(n)$ is the Kronecker impulse function used to represent the onset of a plunge initial condition in discretetime. Note that this model description can also be modified for use in analysis of data which contains both an initial condition and exogenous input by simply including a Kronecker impulse function in Eqn. 13 or neither (time-series analysis) by removing the exogenous input term. 

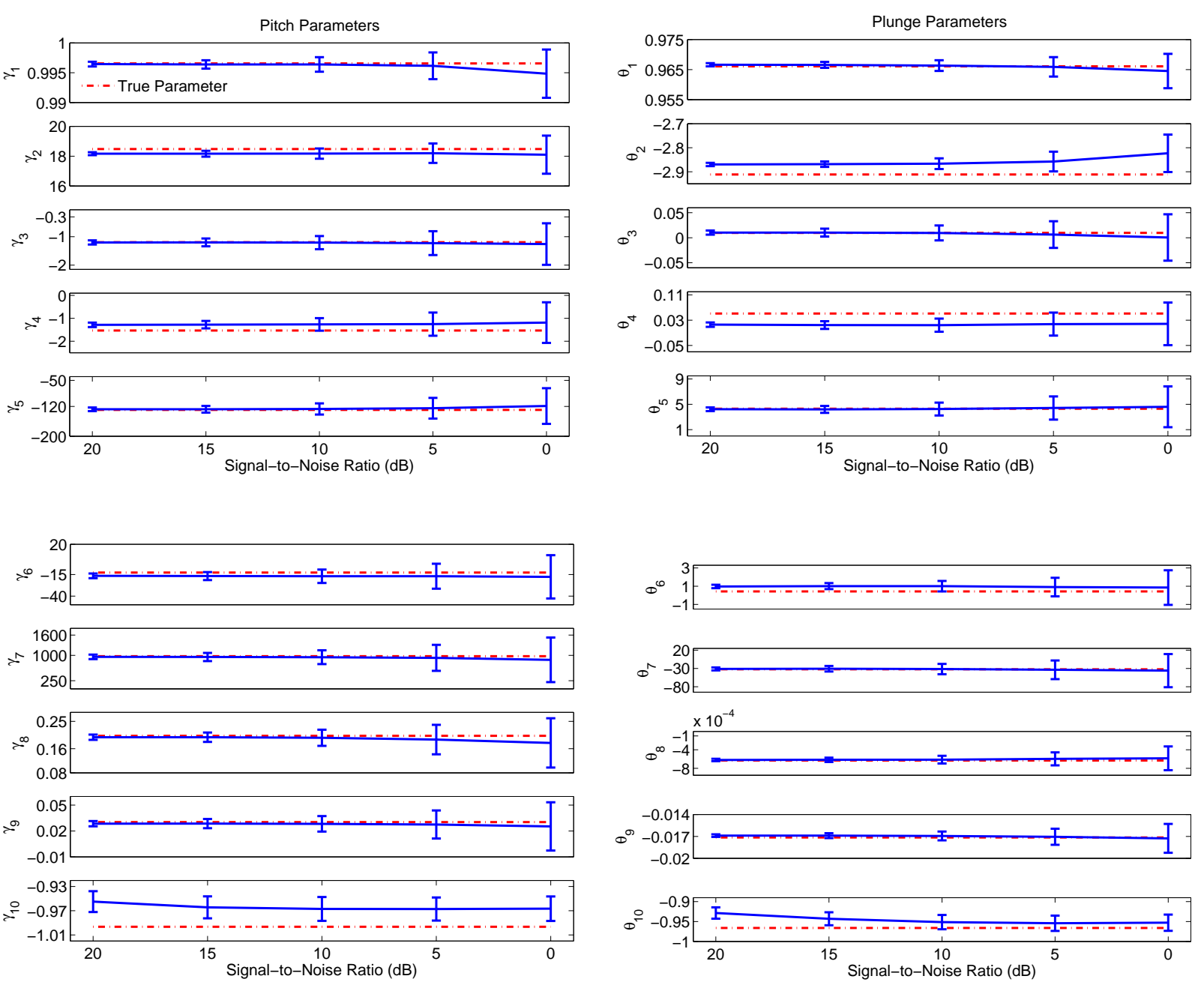

Figure 3. Monte-Carlo study of pitch-plunge NARMAX model parameters. Pitch Parameters (Left): Mean and STD. Plunge Parameters (Right): Mean and STD. 5 Hz chirp input, Gaussian, white, zero-mean noise and N=3,000. Ordinate: STD about mean. Abscissa: Output SNR= 20, 15, 10, 5 and 0 dB. (Note that the abscissa is shown in decreasing SNR which corresponds to increasing noise intensity.)

\section{VII.A. Apparatus}

Data was collected on a unique wind tunnel test apparatus at the Department of Aerospace Engineering, Texas A\&M University. This $2^{\prime} \times 3^{\prime}$ closed-circuit low-speed wind tunnel allows a wing section to move in two degrees-of-freedom and can translate (plunge) and rotate (pitch). This apparatus allows the study of classical bending-torsion flutter. Structural response of the system is governed by springs attached to cams. Stiffness of the springs and the shape of the cams can be altered to prescribe a wide variety of linear and non-linear structural responses.

\section{VII.B. Procedures}

The pitch acceleration was measured by a linear accelerometer, which measured accelerations along one axis. The accelerometer was mounted $0.157 \mathrm{~m}$ from the rotational axis and orthogonal to the $y$-direction (forward-aft) when the airfoil was at a zero angle of attack, giving no acceleration in the $y$-direction. However, a small portion of the plunge acceleration was detected when the airfoil was deflected. The elastic axis was 3/10th of the chord length forward of the midchord.

The free stream velocity was increased in increments of $2 \mathrm{~m} / \mathrm{s}$, from $4 \mathrm{~m} / \mathrm{s}$ to $22 \mathrm{~m} / \mathrm{s}$. Aeroelastic responses were recorded for 45 seconds whilst the free stream velocity was held constant. Flutter was observed to be induced at about $13.5 \mathrm{~m} / \mathrm{s}$. Pitch and plunge displacements and accelerations of this aeroelastic system were sampled at $525 \mathrm{~Hz}$. 

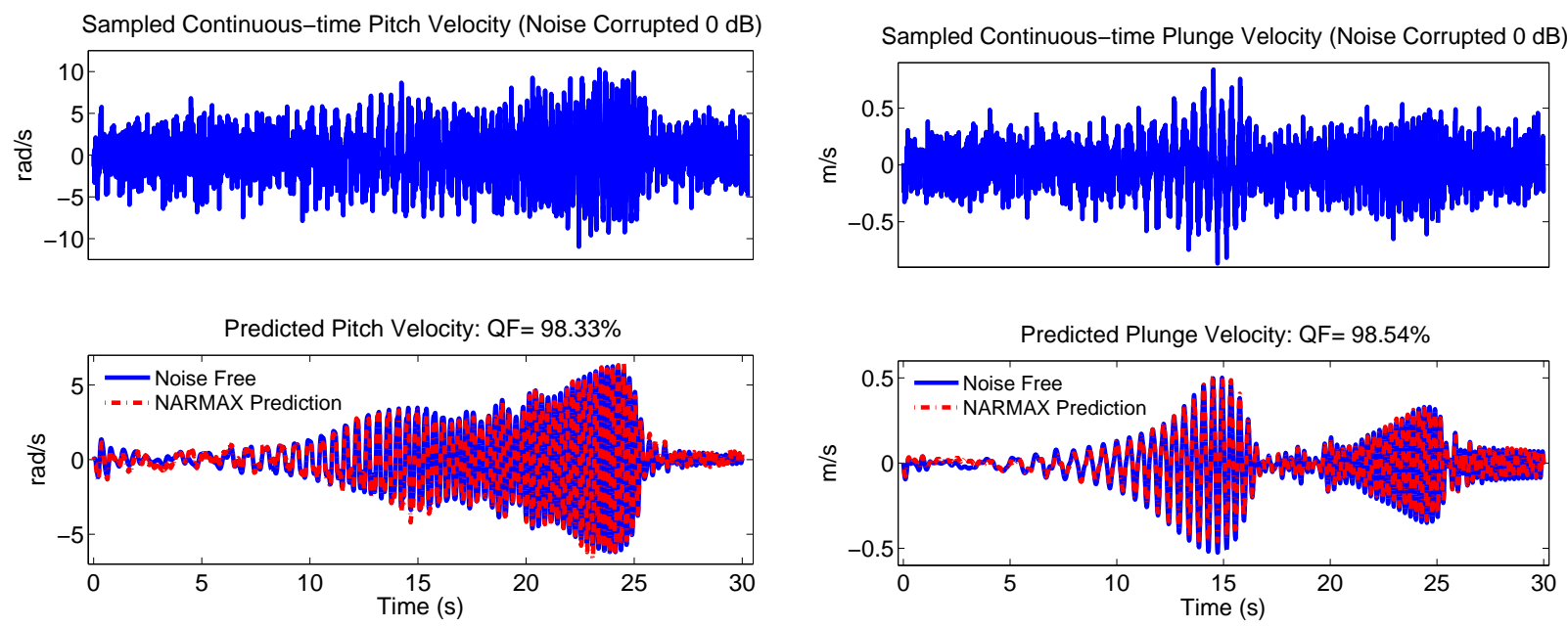

Figure 4. Cross-validation for typical identified NARMAX pitch (left) and plunge (right) models with $N_{v}=3,000$ and Gaussian, white, zero-mean output additive noise (0 dB SNR). Top panels: Measured outputs used for estimation. Bottom panels: Predicted output superimposed on top of noise free output.

After recording, the experimental data was decimated by a factor of 2, resulting in a final sampling rate of 262.5 Hz. The system was identified using the NARMAX approach, as outlined in \$VI.A. except that $N=15,600$ points was used for estimation and $N_{v}=7,800$ points was used for validation.

\section{VII.C. Results}

The results of identifying 10 trials of wing section experiments are presented. Fig. 5(a-b) shows a typical pitch-plunge displacement and velocity trial used for this analysis. The data represents pitch and plunge displacement and velocity sequences whilst the free stream velocity in the wind tunnel was held constant at $16 \mathrm{~m} / \mathrm{s}$. The characteristics of this trial are consistent with those reported in previous work ${ }^{33}$ The lower panels of Fig. 5 (c-d) display a five second slice of the cross-validation (predicted) outputs superimposed on top of the measured outputs, for this trial. The predicted outputs matched the measured outputs with over $98 \%$ QF.

Fig. 6 shows the cross-validation $\% \mathrm{QF}$ for each trial. The results show that the predicted outputs, for these parameter estimates, account for a large portion of the variance. For pitch velocity, the range of $\% \mathrm{QF}$ is from a minimum of $99.73 \%$ to a maximum of $99.98 \%$. For plunge velocity, the range of $\% \mathrm{QF}$ is from a minimum of $98.09 \%$ to a maximum of $99.83 \%$. From the 10 trials examined for this study, $80 \%$ of predicted outputs accounted for more than $99 \% \mathrm{QF}$ of the measured output for both pitch and plunge velocity. This indicates that the NARMAX parameters explain the measured data well. Moreover, for every data set the STD of each model parameter was computed at the 95\% confidence level. These results showed that the STDs did not contain zero. This suggests that the estimated models are accurate. A model parameter whose STD encompasses zero may indicate a spurious model term and, hence, should be reformulated. 34

\section{Discussion}

\section{VIII.A. NARMAX Representation of Pitch-Plunge Velocity Dynamics}

The theoretical results demonstrate that the non-linear difference equation description for the pitch-plunge models are NARMAX models. Simulation results show that the NARMAX models match the continuous-time response well. This suggests that parametric non-linear model forms such as the NARMAX class can be used for modeling aerospace systems.

Non-linear models have the advantage that they cover a wider range of system dynamics than linear models which could allow for slower envelope expansion. Using non-linear models to characterise aeroelastic phenomena can provide significant time and cost savings for test and development of aerospace vehicles. Moreover, the discrete non-linear models of pitch-plunge provide excellent predictions which could be used for control synthesis and statistical studies 

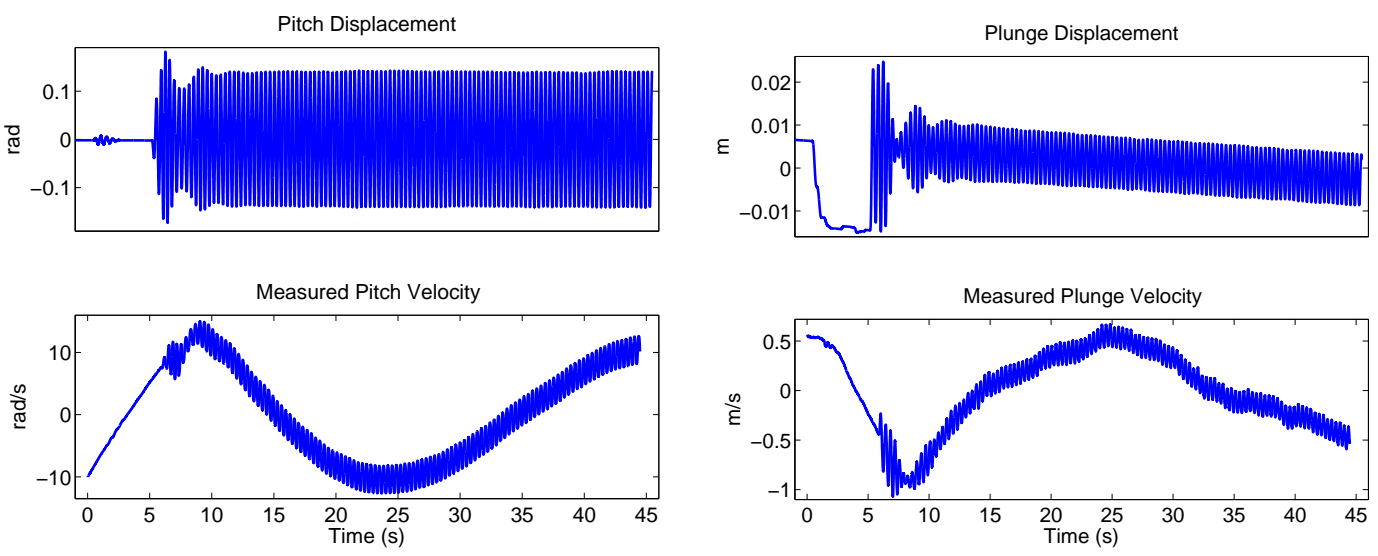

(a)

(b)
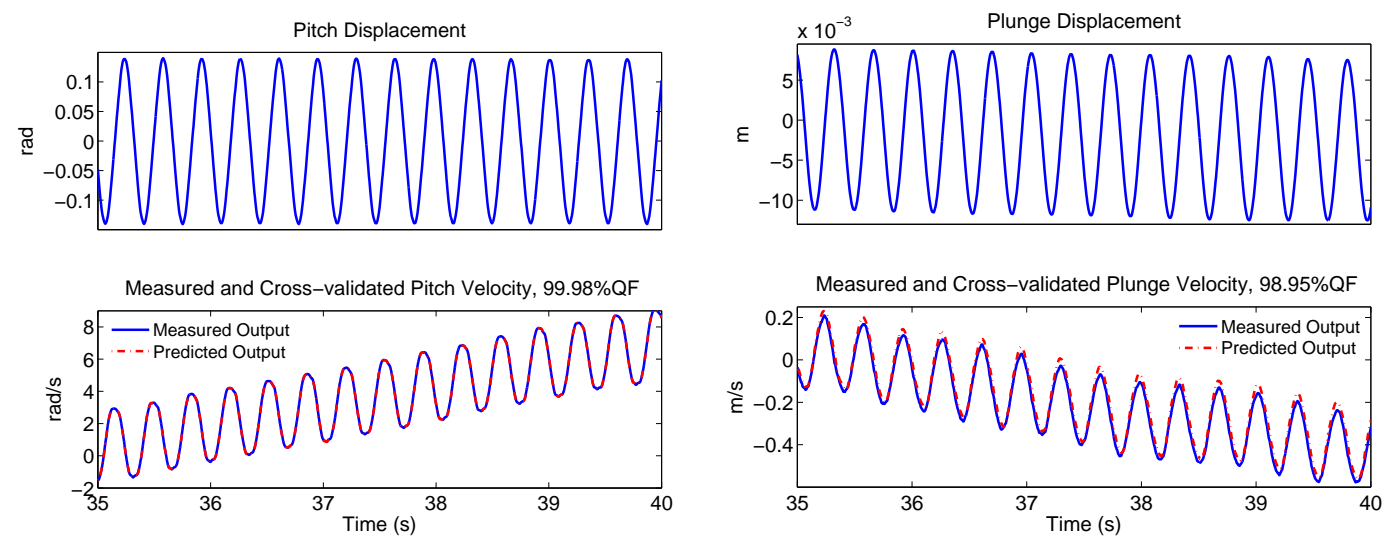

(c)

(d)

Figure 5. a-b (Upper and lower panels): Typical recorded pitch and plunge displacement and velocity. c-d Cross-validation: Upper panels: Five second slice of pitch and plunge displacement. Lower panels: Five second slice of predicted pitch and plunge velocity outputs superimposed on top of measured velocity output for identified NARMAX pitch-plunge velocity models for experimental data set with $N_{v}=7,800$.

of NARMAX coefficients may be of direct relevance for health monitoring of aerostructures.

\section{VIII.B. Discrete-Time Parameter Estimation of Simulated Aeroelastic System}

Simulation studies in $\$$ VI.A showed that, for a NARMAX model representation, the mean of Monte-Carlo estimates for NARMAX parameters matched the theoretical values well for all SNR levels. However, estimates of some parameters, e.g. $\gamma_{10}$, did not correspond well to theoretically computed values. As stated earlier, lagged error terms are difficult to identify accurately. Error terms represent a stochastic process which cannot be measured. This stochastic process is approximated by a deterministic signal of prediction errors which is only a (poor) estimate of the noise. $\frac{28,22}{2}$

\section{VIII.C. Identification of Experimental Aeroelastic Data}

High $\%$ QF cross-validation fits obtained for parameter estimates using NARMAX identification methods (see Fig. 6) shows that the identified parameters explain the experimental data well. Using $\% \mathrm{QF}$ alone as an indicator of model goodness may lead to incorrect interpretations of model validity. However, in many cases for non-linear models this may be the only indicator that is readily available.

A model validation technique for non-linear systems, using higher-order correlations, was developed by Billings 


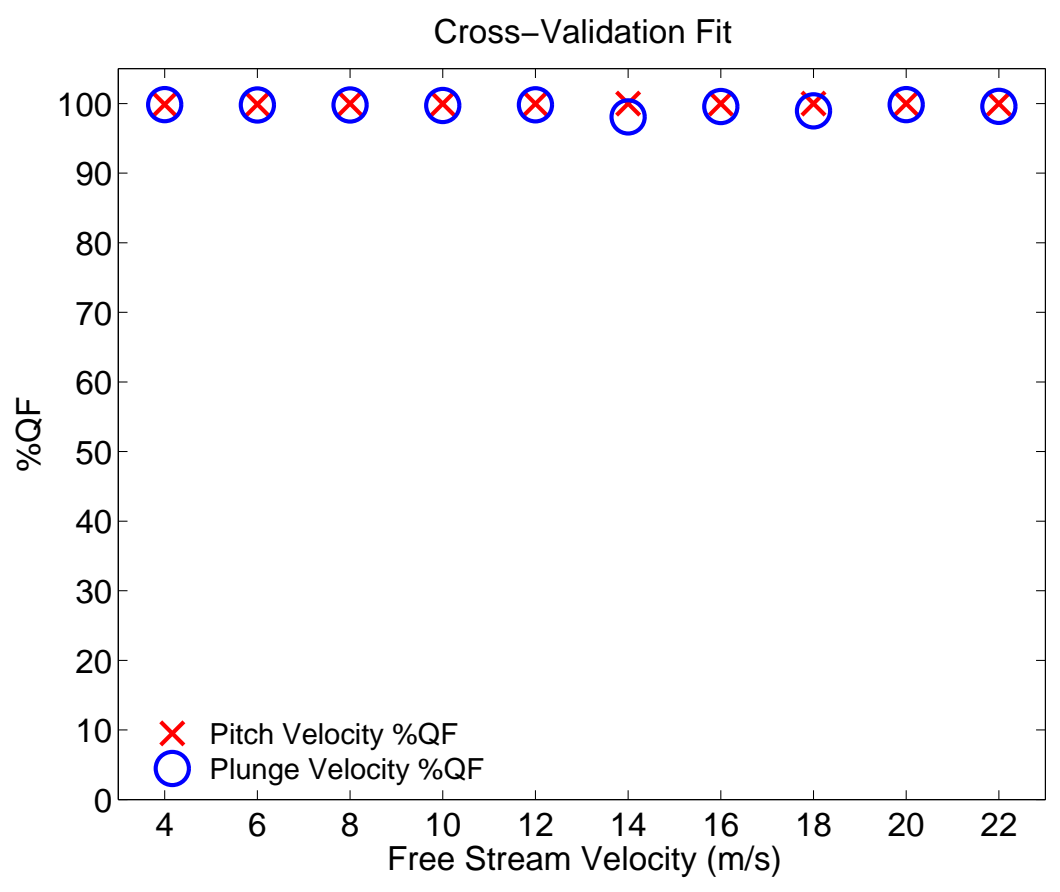

Figure 6. Cross-validation. \%QF vs. free stream velocity (experimental trial).

and Voon $\sqrt[35,36]{ }$ Korenberg and Hunter ${ }^{37}$ showed that this model validation technique fails for simple cases. Therefore, this approach was not implemented in favor of using the $\% \mathrm{QF}$ alone as an indicator of model goodness.

When studying aeroelastic systems it may not be practical to assume that the exact model order and structure are well known a priori. In aerospace systems analysis one of the main objectives is not only to estimate system parameters but to gain insight into the structure of the underlying system. It would be worthwhile in a future study to investigate what happens if NARMAX structure detection methods $38,39,40,34$ are allowed to analyse the data to find the best structure from the data set. This may then indicate deficiencies in the analytical model and could lead to improved modeling strategies.

\section{Conclusions}

Theoretical results demonstrate that the non-linear difference equation description for the pitch-plunge model is a NARMAX model. Simulation results show that the NARMAX model matches the continuous-time response well. Moreover, this paper contributes to the understanding of the use of parametric identification techniques for modeling of non-linear aerospace systems. The main point here is that the NARMAX form is clearly amenable to the study of a wide range of aerospace systems, and could be computationally efficient. NARMAX modeling and identification techniques should be examined further especially in the case of severe non-linear behavior.

\section{References}

${ }^{1}$ Lee, B., Pricei, S., and Wong, Y., "Nonlinear aeroelastic analysis of airfoils: bifurcation and chaos," Progress in Aerospace Sciences, Vol. 35, No. 3, 1999, pp. 205-334.

${ }^{2}$ Bunton, R. and Denegri, C., "Limit Cycle Oscillation Characteristics of Fighter Aircraft," AIAA Journal of Aircraft, Vol. 37, No. 5, 2000, pp. 916-918. 1727.

${ }^{3}$ Chen, P., Sarhaddi, D., and Liu, D., "Limit Cycle Oscillation Characteristics of Fighter Aircraft with External Stores," AIAA-98, 1998, p.

${ }^{4}$ Denegri, C., "Limit Cycle Oscillation Flight Test Results of a Fighter with External Stores," AIAA Journal of Aircraft, Vol. 37, No. 5, 2000, pp. 761-769.

${ }^{5}$ Greblicki, W. and Pawlak, M., "Nonparametric Identification of a Cascade Nonlinear Time Series System," Signal Processing, Vol. 22, No. 1, 1991, pp. 61-75.

${ }^{6}$ Kosut, R., Lau, M., and Boyd, S., "Set-membership Identification of Systems with Parameteric and Nonparametric Uncertainty," IEEE 
Transactions on Automatic Control, Vol. 37, No. 7, 1992, pp. 929-942.

${ }^{7}$ Masri, S. and Caughey, T., "A nonparametric identification technique for nonlinear dynamic problems," Journal of Applied Mechanics, Vol. 46, No. 2, 1979, pp. 433-447.

${ }^{8}$ Lind, R., Prazenica, R., and Brenner, M., "Estimating Nonlinearity Using Volterra Kernals in Feedback With Linear Models," Nonlinear Dynamics, Vol. 39, No. 1, January 2005, pp. 3-23.

${ }^{9}$ Leontaritis, I. and Billings, S., "Input-output parametric models for non-linear systems Part I: deterministic non-linear systems," International Journal of Control, Vol. 41, No. 2, 1985, pp. 303-328.

${ }^{10}$ Leontaritis, I. and Billings, S., "Input-output parametric models for non-linear systems Part II: stochastic non-linear systems," International Journal of Control, Vol. 41, No. 2, 1985, pp. 329-344.

${ }^{11}$ Billings, S. and Chen, S., "Extended Model Set, Global Data and Threshold Model Identification of Severely Non-linear Systems," International Journal of Control, Vol. 50, No. 5, 1989, pp. 1897-1923.

${ }^{12}$ Chen, S. and Billings, S., "Representations of non-linear systems: the NARMAX model," International Journal of Control, Vol. 49, No. 3 , 1989, pp. 1013-1032.

${ }^{13}$ Chen, S., Billings, S., Cowan, C., and Grant, P., "Practical identification of NARMAX models using radial basis functions," International Journal of Control, Vol. 52, No. 6, 1990, pp. 1327-1350.

${ }^{14}$ Kukreja, S., Galiana, H., and Kearney, R., "NARMAX Representation and Identification of Ankle Dynamics," IEEE-TBME, Vol. 50, No. 1, 2003, pp. 70-81.

${ }^{15}$ O'Neil, T. and Strganac, T. W., "Nonlinear Aeroelastic Response - Analyses and Experiments," AIAA-96, 1996, p. 0014.

${ }^{16}$ O'Neil, T., Gilliatt, H. C., and Strganac, T. W., "Investigations of Aeroelastic Response for a System with Continuous Structural Nonlinearities," AIAA-96, 1996, p. 1390.

${ }^{17}$ Dowell, E., "Nonlinear Aeroelasticity," Structures, Structural Dynamics, and Materials Conference, Vol. 31, 1990, pp. 1497-1509.

${ }^{18}$ Tang, D. and Dowell, E., "Flutter and Stall Response of a Helicopter Blade with Structural Nonlinearity," AIAA Journal of Aircraft, Vol. 29, 1990, pp. 953-960.

${ }^{19}$ Yang, Z. and Zhao, L., "Analysis of Limit Cycle Flutter of an Airfoil in Incompressible Flow," Journal of Sound \& Vibration, Vol. 123, No. 1, 1988, pp. 1-13.

${ }^{20}$ Zhao, L. and Yang, Z., "Chaotic Motions of an Airfoil with Nonlinear Stiffness in Incompressible Flow," Journal of Sound \& Vibration, Vol. 138, No. 2, 1990, pp. 245-254.

${ }^{21}$ Franklin, G., Powell, J., and Emami-Naeini, A., Feedback Control of Dynamic Systems, Addison-Wesley, New York, 4th ed., 2002.

${ }^{22}$ Ljung, L., System Identification: Theory for the User, Prentice Hall, Inc., Upper Saddle River, New Jersey, 2nd ed., 1999.

${ }^{23}$ Åström, K. and Wittenmark, B., Computer-Controlled Systems: Theory and Design, Prentice Hall, Englewood Cliffs NJ, 3rd ed., 1996.

${ }^{24}$ Panuska, V., "A Stochastic Approximation Method for Identification of Linear Systems Using Adaptive Filtering," Proceedings 9th Joint Automatic Control Conference, IEEE, Ann Arbor, MI, USA, June 1968, pp. 1014-1021.

${ }^{25}$ Panuska, V., "An Adaptive Recursive Least Squares Identification Algorithm," Proceedings 8th IEEE Symp. on Adaptive Processes, University Park, PA, USA, Nov. 1969, p. paper 6e.

${ }^{26}$ Young, P., "The Use of Linear Regression and Relaxed Procedures for the Identification of Dynamic Processes," Proceedings 7th IEEE Symp. on Adaptive Processes, Los Angeles, CA, USA, Dec. 1968, pp. 501-505.

${ }^{27}$ Billings, S. and Voon, W., "Least squares parameter estimation algorithms for non-linear systems," International Journal of Systems Science, Vol. 15, No. 6, 1984, pp. 601-615.

${ }^{28}$ Goodwin, G. and Payne, R., Dynamic System Identification: Experiment Design and Data Analysis, Vol. 136 of Mathematics in Science and Engineering, Academic Press, New York, 1977.

${ }^{29}$ Walter, E. and Pronzato, L., Identification of Parametric Models, Springer-Verlag, Berlin, Germany, 1st ed., 1997.

${ }^{30}$ Söderström, T. and Stoica, P., System Identification, Prentice-Hall International, Piscataway, New Jersey, 1989.

${ }^{31}$ Stoica, P. and Söderström, T., "Asymptotic behavior of some bootstrap estimators," International Journal of Control, Vol. 33, No. 3, 1981, pp. $433-454$

${ }^{32}$ Shao, J., "Linear model selection by cross-validation," Journal of the American Statistical Association, Vol. 88, No. 422, 1993 , pp. $486-494$.

${ }^{33}$ Kurdila, A., Prazenica, R., Rediniotis, O., and Strganac, T., "Multiresolution Methods for Reduced-Order Models for Dynamical Systems," AIAA Journal of Guidance, Control, and Dynamics, Vol. 24, No. 2, 2001, pp. 193-200.

${ }^{34}$ Kukreja, S., Galiana, H., and Kearney, R., "A Bootstrap Method for Structure Detection of NARMAX Models," International Journal of Control, Vol. 77, No. 2, 2004, pp. 132-143.

${ }^{35}$ Billings, S. and Voon, W., "Structure detection and model validation tests in the identification of nonlinear systems," IEE Proceedings, Part D-Control Theory and Applications, Vol. 130, No. 4, 1983, pp. 193-199.

${ }^{36}$ Billings, S. and Voon, W., "Correlation based model validity tests for non-linear models," International Journal of Control, Vol. 44, No. 1, 1986, pp. 235-244.

${ }^{37}$ Korenberg, M. and Hunter, I., "The identification of nonlinear biological systems: Wiener kernel approaches," Annals of Biomedical Engineering, Vol. 18, 1990, pp. 629-654.

${ }^{38}$ Korenberg, M., "Orthogonal Identification of Nonlinear Difference Equation Models," Proceedings of the Midwest Symposium on Circuit Theory, Vol. 1, 1985, pp. 90-95.

${ }^{39}$ Korenberg, M., Billings, S., Liu, Y., and Mcllroy, P., "Orthogonal parameter estimation algorithm for non-linear stochastic systems," International Journal of Control, Vol. 48, No. 1, 1988, pp. 193-210.

${ }^{40}$ Kukreja, S., Kearney, R., and Galiana, H., "A Bootstrap Method for NARMAX Model Order Selection," Proc. 4th IFAC Symposium on Modelling and Control in Biomedical Systems, Vol. 4, Karlsburg/Greifswald, Germany, March 2000, pp. 353-356. 